\title{
Nucleic Acid Content of HBV Capsids Depends on its CTD-Phosphorylation State
}

Gökhan Tolun ${ }^{1}$, Naiqian Cheng ${ }^{1}$, Bernard J. Heymann ${ }^{1}$, Paul T. Wingfield ${ }^{1}$, Laurie Ludgate ${ }^{2}$, Jianming $\mathrm{Hu}^{2}$ and Alasdair C. Steven ${ }^{1}$

${ }^{1 .}$ National Institute of Arthritis and Musculoskeletal and Skin Diseases at National Institutes of Health, Bethesda, MD, USA.

2. Department of Microbiology and Immunology, The Penn State University College of Medicine, Hershey, Pennsylvania, USA.

HBV infections result in fatal conditions such as cirrhosis and liver cancer in 15 to $25 \%$ of chronic patients. Considering a global estimation of 350 million chronic HBV infections [1], understanding the HBV life cycle and developing remedies against it remains a vital task. During its replication cycle, HBV packs a pregenomic RNA (pgRNA) into its capsid, which is first converted to a full-length ssDNA, and then to a partially double-stranded DNA (dsDNA) genome, while the pgRNA itself is degraded. HBV capsid is formed by the viral capsid protein (Cp) composed of an N-terminal domain (residues 1-149) that forms the outer shell, and an arginine-rich C-terminal domain (CTD, residues 150183) that lines the capsid. The CTD is known to be involved in pgRNA packaging, and particularly, phosphorylation of the serine residues in the three SPRRR repeats was shown to be important [2]. The mechanistic details of these processes are still unknown.

We examined the structures of three HBV capsid populations by cryoEM. Two Cp constructs, wild-type (wt-1) and a mutant form with three phosphorylation-target serines at positions 155, 162, and 170 mutated to alanines (3A) were expressed in HEK293T cells to generate capsids. A third capsid preparation (wt-2) was made by co-expressing in HepG-2 cells the wt Cp construct together with the viral polymerase and pgRNA. The capsids were purified and vitrified for observation. Images were acquired on film using a Philips CM200-FEG electron microscope at $120 \mathrm{kV}$. 3D image reconstructions were calculated from the scanned micrographs, using Bsoft.

Representative fields of capsids are shown in Figure 1 and central slices of the reconstructions in Figure 2. The resolutions of the wt-1,3A and wt-2 maps are 9.6, 11.5 and $10 \AA$ respectively (FSC 0.5). The reconstructions differ mainly in the intensity of the ring of density, peaking at a radius of $\sim 105 \AA$, that underlies the capsid wall. The overall density distribution within this ring is similar to the RNA densities observed previously $[3,4]$. The $3 \mathrm{~A}$ capsids have $\sim 2.7$ fold more density in this ring than either wt sample, indicative of a larger amount of nucleic acids packaged. The amount of RNA packaged by these wt capsids assembled in mammalian cells is significantly less than when the same $\mathrm{Cp}$ construct is assembled in E. coli [4]. These observations suggest that phosphorylation of the three Ser residues reduces the amount of RNA packaged. When no phosphorylation takes place, as in the $3 \mathrm{~A}$ mutant or in E. coli where the kinase responsible is presumably lacking [5], more RNA is packaged. When the capsids were run in an agarose gel and stained for nucleic acids, the resulting molecular weight corresponded mostly to tRNAs, suggesting that it is the nonspecific packaging that increases in the absence of phosphorylation. Previously, the 3A mutant was reported to suppress pgRNA encapsidation [6]. Here, we show a possible reason for this observation: the pgRNA packaging defect is probably not due to the loss of RNA packaging activity per se, but due to losing the ability to differentiate pgRNA from other RNAs, such as tRNAs [7]. 


\section{References:}

[1] CDC. Hepatitis B FAQs for Health Professionals. http://www.cdc.gov/hepatitis/HBV/HBVfaq.htm.

[2] Wei, Y., et al., Pathol Biol (Paris) 58 (2010), p. 267-72.

[3] Wang, J.C., M.S. Dhason, and A. Zlotnick, PLoS Pathog 8 (2012), p. e1002919.

[4] Zlotnick, A., et al., Proc Natl Acad Sci U S A 94 (1997), p. 9556-61.

[5] Ludgate, L., et al., J Virol 86 (2012), p. 12237-50.

[6] Gazina, E.V., et al., J Virol 74 (2000), p. 4721-8.

[7] This work is supported by the Intramural Research Program of NIAMS and NIH grant R01AI043453 to J.H.
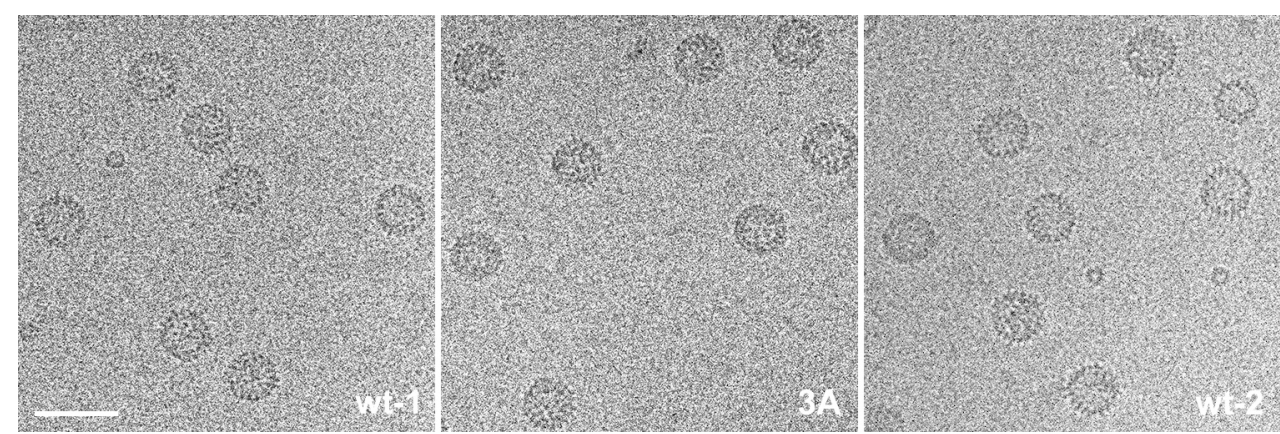

Figure 1. Representative raw micrographs of the three samples used. Scale bar is $500 \AA$.
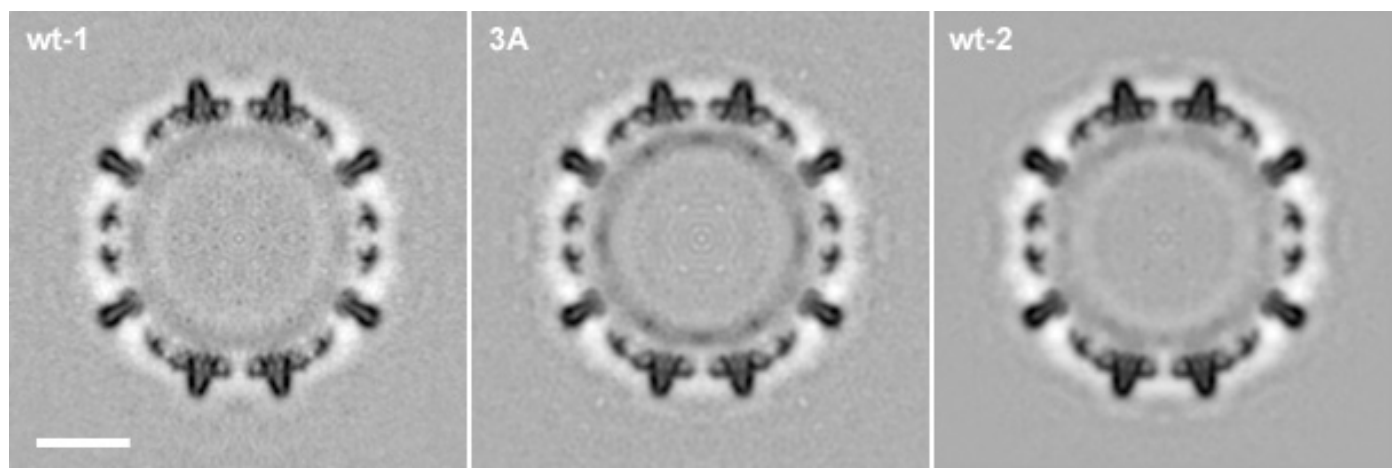

Figure 2. Center slices of the reconstructions of three HBV capsid populations. Scale bar is $100 \AA$.

wt-1

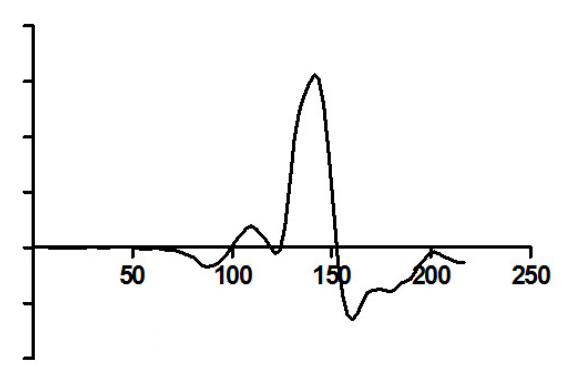

$3 A$

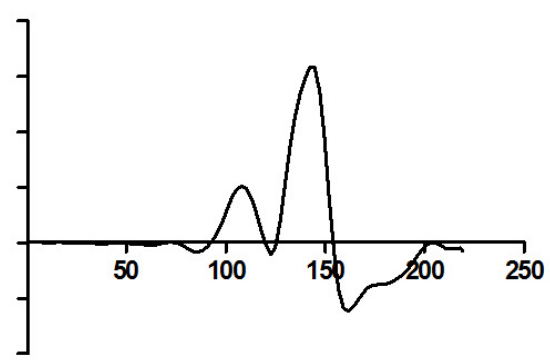

wt-2

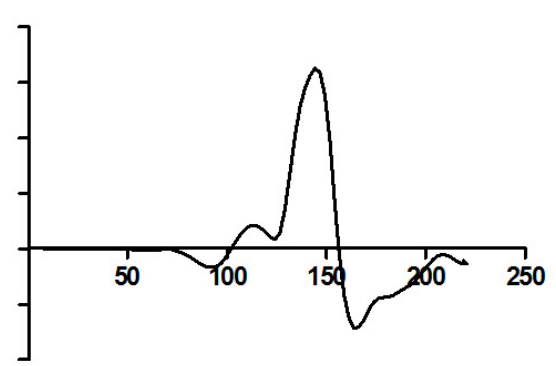

Figure 3. Radial density profiles of the reconstructions shown above. $\mathrm{X}$ axis is distance from the center of the volume in pixels, and $\mathrm{Y}$ axis is the total density in arbitrary units calculated by adding the voxel values at the radius corresponding to the $\mathrm{X}$ value. 